UdeM-LPS-TH-93-150, hep-th/9306027

\title{
Spontaneous Symmetry Breaking and the Renormalization of the Chern-Simons Term
}

\author{
Avinash Khare, ${ }^{*}$ R. B. MacKenzie, P. K. Panigrahi ${ }^{\dagger}$ and M. B. Paranjape \\ Laboratoire de Physique Nucléaire, Université de Montréal C.P. 6128, succ. centreville, \\ Montréal, Québec, Canada, H3C 3J7
}

\begin{abstract}
We calculate the one-loop perturbative correction to the coefficient of the Chern-Simons term in non-abelian gauge theory in the presence of Higgs fields, with a variety of symmetry-breaking structures. In the case of a residual $U(1)$ symmetry, radiative corrections do not change the coefficient of the ChernSimons term. In the case of an unbroken non-abelian subgroup, the coefficient of the relevant Chern-Simons term (suitably normalized) attains an integral correction, as required for consistency of the quantum theory. Interestingly, this coefficient arises purely from the unbroken non-abelian sector in question; the orthogonal sector makes no contribution. This implies that the coefficient of the Chern-Simons term is a discontinuous function over the phase diagram of the theory.
\end{abstract}

*permanent address: Institute of Physics, Sachivalaya Marg, Bhubaneswar, 751005, India.

†permanent address: Department of Physics, University of Hyderabad, Hyderabad, 500134, India. 
Yang Mills theories in $2+1$ dimensions have attracted much attention in recent years. This is primarily because of the possibility of adding a new topological term to the action, the Chern-Simons (CS) term [1.2], which has had diverse applications from condensed matter physics to pure mathematics. The possibility of particles obeying arbitrary statistics, anyons [3], can be elegantly formulated by using a CS term [G]. Anyons are known to play an important role in the fractional quantum Hall effect [5], and provide a mechanism for superconductivity [6]. The limit where the action is the pure CS term [7] results in a topological field theory [8]. The only observables of this theory are Wilson loops, whose expectation values give rise to knot invariants. Considering such a theory with a non-compact gauge group, for example the $2+1$ dimensional Poincare group, gives a consistent quantum theory of $2+1$ dimensional gravity [9]. Three-dimensional field theories are the high temperature limits of corresponding $3+1$ dimensional field theories [10]. Thus parity violating theories in 3+1 dimensions, like the Standard Model, will in general contain the CS term in their effective actions in the high temperature limit. Therefore it is interesting to study $2+1$ dimensional Yang Mills theory with the CS term.

For a non-abelian gauge theory with gauge group $\mathcal{G}$, the CS action, although invariant under small gauge transformations, changes by an integer multiple of $8 \pi^{2} \mu / g^{2}$ under a large gauge transformation, where $\mu$ is the coefficient of the CS term and $g$ is the gauge coupling constant. This leads to the celebrated quantization condition [2]

$$
q \equiv \frac{4 \pi \mu}{g^{2}}=n, \quad n=0, \pm 1, \pm 2, \cdots
$$

in order to maintain the invariance of $e^{i S}$.

An important question to address is whether this quantization condition is respected by quantum corrections. This issue was considered by Pisarski and Rao [11], for the case of a pure gauge theory with dynamics governed by the usual Yang Mills term and the CS term. They found that the quantization condition is indeed preserved to one loop; however, the integer on the R.H.S. of $(\mathbb{1})$ is shifted by $N$ for $\mathcal{G}=S U(N)$. This calculation has been extended to two loops by Giavirini, et al. [12], who found no further correction in the limit 
of pure CS interaction, confirming the expectation that there are no corrections beyond one loop in that theory [11].

Subsequently, the question of quantization was considered for the case of a completely spontaneously broken gauge theory by Khlebnikov and Shaposhnikov [13]. They found that at the one loop level $q$ is multiplicatively renormalized by a complicated function of the three mass scales in the problem ( $\mu$, the symmetry breaking mass scale and the physical Higgs mass scale), and violates (四).

At first sight, this result indicates loss of gauge invariance and inconsistency of the theory. However, the situation is not as catastrophic as it first appears: the underlying theory can continue to maintain gauge invariance, as manifested for example in the effective action. The effective action would be a completely gauge invariant functional of external gauge and scalar fields, but would cease to appear this way when the scalar field is evaluated at its vacuum expectation value. Indeed, it was observed in 13 that in the presence of spontaneous symmetry breaking, other terms exist in the effective action which reduce to the CS term when $\phi \rightarrow\langle\phi\rangle=v$, but which are nonetheless invariant under large gauge transformations. If this is the case, the calculation in [13] is not, in fact, a calculation of the coeffecient of the CS term alone. Rather, it is the sum of the coeffecients of the CS term and the other terms which reduce to it. This leaves the possibility, which should be verified, that the non-quantized result obtained in [13] is the sum of a quantized CS coeffecient and non-quantized (yet perfectly acceptable) contributions from the other terms.

In a slightly different context (namely, the spontaneously-broken abelian case, where quantization of the coefficient of the CS term is not required for consistency), a complicated radiatively induced correction to (apparently) the CS term [14] was found to be due to other terms in the effective action which reduce to the CS term in the symmetry-breaking phase, exactly as described above: the coefficient of the CS term itself is in fact unchanged in that model [15].

In this work, we consider the case of partial breaking of a non-abelian gauge symmetry. For an abelian unbroken subgroup, this is a proving ground for the Coleman-Hill theorem 
[16]. The theorem asserts that if there are no massless states in the theory other than the photon, and if there is manifest Lorentz invariance, then the renormalization of the CS term is zero except for the one-loop contribution of fermions. They specifically exclude the case of unbroken non-abelian gauge theories, where each generator forms an abelian subgroup, since there exists no quantization which respects the conditions of the theorem. The case of a partially broken non-abelian gauge theory is more interesting. With symmetry breaking to just $U(1)$, all the remaining gauge bosons attain explicit masses; hence, the Coleman-Hill theorem implies no renormalization of the CS term. On the other hand, for a non-abelian unbroken subgroup $(S U(M)$, where $M<N$, say), the Coleman-Hill theorem does not apply. We must still have, at the very least, a quantized coefficient of the CS term for the gluons of the unbroken subgroup to have a consistent theory [2]. Although there is no formal proof, there is a suggestion in [11] that there might be an equivalent non-abelian version of the Coleman-Hill theorem which states that under suitable general conditions the renormalization of the CS term in $S U(N)$ theories is by $q \rightarrow q+N$, as found in [11]. If this is indeed the case, one might expect that the renormalization of the CS term for an unbroken, non-abelian sector to be that which corresponds to the unbroken subgroup only $(q \rightarrow q+M$ for unbroken $S U(M))$. This is because one can construct a Lorentz-invariant gauge where there are no massless particles outside the unbroken sector, and the conjectured non-abelian version of the Coleman-Hill theorem (assuming its assumptions are those of the abelian version) would imply that no contribution to $q$ will arise from the broken sector.

In the following, we find that exactly this scenario takes place, to one loop.

We begin by studying the gauge group $S U(3)$ spontaneously broken to an $S U(2)$ subgroup. The latter being nonabelian, we must demand the quantization of $q_{S U(2)}$, the value of $q$ for the unbroken $S U(2)$ subgroup, including radiative corrections. (It is important to observe that no terms exist in the effective action which would reduce under spontaneous symmetry breaking to the unbroken CS term.) We find, confirming the work of Chen, et al. [17] and correcting the calculation of the original version of this paper, that $q_{S U(2)} \rightarrow q_{r e n}^{S U(2)}=q_{S U(2)}+2$. 
The coefficient of the CS term remaining quantized in accordance with (11), these results do not uncover any inconsistency in the quantum version of the theory of the type discussed in [2]. Nonetheless, we find the results rather perplexing: the coefficient of the CS term is apparently a discontinuous function over the phase diagram of the theory. Specifically, when the pattern of symmetry breaking changes, the coefficient of the CS term jumps by a discrete amount. Although such behaviour has been seen before, for example in the case of massive fermions where the contribution to the CS term is proportional to the sign of the fermion mass, we do not expect this to arise from scalars.

This behaviour seems to be the rule rather than the exception. Indeed, we have generalized the above to include several different patterns of symmetry breaking, and we find in all cases results consistent with the nonabelian generalization of the Coleman-Hill theorem conjectured above: when $S U(N)$ is broken to a subgroup which contains in general $S U(M)$ and $U(1)$ factors, the renormalization of the CS term for unbroken $U(1)$ subgroups is zero, while that for unbroken $S U(M)$ subgroups is by $\delta q=M$. The calculation in all cases separates into contributions from within the subgroup under consideration and from the orthogonal sector of the theory; the former gives the simple result just stated while the latter gives a vanishing contribution. As for the renormalization of $q$ for the broken sector of the theory, one can, as outlined above, construct terms in the effective action which reduce to the CS term when the scalar field is replaced by its expectation value, and thus the type of calculation undertaken here is insufficient to determine its renormalization.

We begin with the case of $S U(3)$ spontaneously broken to $S U(2)$ via a triplet of Higgs in the fundamental representation. The corresponding Lagrangian is given by

$$
\begin{aligned}
\mathcal{L}= & -\frac{1}{4} F_{a \mu \nu} F_{a}^{\mu \nu}-\frac{\mu}{2} \epsilon_{\mu \nu \lambda}\left(A_{a}^{\mu} \partial^{\nu} A_{a}^{\lambda}+\frac{1}{3} g f_{a b c} A_{a}^{\mu} A_{b}^{\nu} A_{c}^{\lambda}\right) \\
& +\left(D^{\mu} \phi\right)_{A}^{\dagger}\left(D_{\mu} \phi\right)_{A}+m^{2}\left(\phi_{A}^{\dagger} \phi_{A}\right)-\lambda\left(\phi_{A}^{\dagger} \phi_{A}\right)^{2}
\end{aligned}
$$

where

$$
\begin{aligned}
& F_{a \mu \nu}=\partial_{\mu} A_{a \nu}-\partial_{\nu} A_{a \mu}+g f_{a b c} A_{b \mu} A_{c \nu} \\
& \left(D_{\mu} \phi\right)_{A}=\partial_{\mu} \phi_{A}-i g \frac{\lambda_{A B}^{a}}{2} A_{a \mu} \phi_{B}
\end{aligned}
$$


and $\lambda_{A B}^{a}$ are the Gell-Mann matrices with $f_{a b c}$ being the structure constants. The choice of the Higgs potential implies a non-zero vacuum expectation value for $\phi$. We write $\phi=\phi^{\prime}+\langle\phi\rangle_{0}$ with

$$
\langle\phi\rangle_{0}=\left(\begin{array}{c}
0 \\
0 \\
\frac{v}{\sqrt{2}}
\end{array}\right), \quad v=\sqrt{\frac{m^{2}}{\lambda}} .
$$

The gauge fixing and ghost terms are given by

$$
\begin{aligned}
\mathcal{L}^{\prime}= & -\frac{1}{2 \xi}\left(\partial_{\mu} A_{a}^{\mu}-i g \xi\left(\langle\phi\rangle_{0}^{\dagger} \frac{\lambda^{a}}{2} \phi^{\prime}-\phi^{\prime \dagger} \frac{\lambda^{a}}{2}\langle\phi\rangle_{0}\right)\right)^{2} \\
& +\partial_{\mu} \bar{\eta}_{a} \partial^{\mu} \eta_{a}-i g f_{a b c} \partial_{\mu} \bar{\eta}_{a} A_{b}^{\mu} \eta_{c} .
\end{aligned}
$$

The vertices are standard for a spontaneously broken non-abelian gauge theory; however, the CS term introduces an extra (parity odd) three-gluon vertex. The gluon propagator is, however, more involved. It is colour diagonal; for colour indices in the unbroken sector, $(a=1,2,3)$, in Landau gauge $(\xi=0)$, it is given by

$$
i \Delta_{\mu \nu}=-i \frac{\left(g_{\mu \nu}-\frac{k_{\mu} k_{\nu}}{k^{2}}\right)-i \mu \epsilon_{\mu \nu \lambda} \frac{k^{\lambda}}{k^{2}}}{k^{2}-\mu^{2}}
$$

while for the broken generators we have 11, 18

$$
i \Delta_{\mu \nu}=-i \frac{\left(g_{\mu \nu}-\frac{k_{\mu} k_{\nu}}{k^{2}}\right)\left(k^{2}-m_{W}^{2}\right)-i \mu \epsilon_{\mu \nu \lambda} k^{\lambda}}{\left(k^{2}-m_{+}^{2}\right)\left(k^{2}-m_{-}^{2}\right)} .
$$

Here

$$
m_{ \pm}=\sqrt{m_{W}^{2}+\frac{\mu^{2}}{4}} \pm \frac{\mu}{2}
$$

and $m_{W}$ is the contribution to the gluon mass from the Higgs mechanism. We have $m_{W}=$ $v g / 2 \equiv m_{D}$ for the iso-doublet massive vectors $(a=4,5,6,7)$ and $m_{W}=v g / \sqrt{3} \equiv m_{S}$ for the iso-singlet massive vector $(a=8)$.

Following Pisarski and Rao [1]1], we calculate $q_{\text {ren }}$ according to

$$
q_{\text {ren }}=\frac{4 \pi \mu}{g^{2}} Z_{m} \tilde{Z}^{2}
$$

to one loop in Landau gauge, for the unbroken $S U(2)$ subgroup. Here $Z_{m}$ and $\tilde{Z}$ are, respectively, the renormalization constants for the odd part of the gluon self-energy and the 
ghost self-energy. We note that $q_{\text {ren }}$ for the broken generators will be different than that for the unbroken $S U(2)$ subgroup since the physical Higgs contributes to $Z_{m}$ in this case. We do not present the details of the calculation since it is amply described in [17]. For $q_{r e n}^{S U(2)}$, the renormalized $q$ for the unbroken $S U(2)$ subgroup, we must find $Z_{m}$ and $\tilde{Z}$ for that sector. Beyond those calculated in [11], there are additional contributions, $\delta Z_{m}$, to the gluon self-energy coming from the massive iso-doublet vector bosons circulating in the gluon loop and from the loop containing unphysical scalars. The ghost self-energy is also augmented by an additional contribution $\delta \tilde{Z}$, from the loop containing massive iso-doublet vector bosons. The massive iso-singlet vector boson actually does not contribute at this order. We find

$$
\begin{aligned}
\delta Z_{m} & =g^{2} \int \frac{d^{3} p}{(2 \pi)^{3}}\left(-2 \frac{\left(p^{2}+m_{D}^{2}\right)^{2}}{\left(p^{2}+m_{+}^{2}\right)^{2}\left(p^{2}+m_{-}^{2}\right)^{2}}+\frac{16}{3} \frac{p^{2}\left(p^{2}+m_{D}^{2}\right)}{\left(p^{2}+m_{+}^{2}\right)^{2}\left(p^{2}+m_{-}^{2}\right)^{2}}\right. \\
& \left.-\frac{2}{3} \frac{\mu^{2}\left(p^{2}+m_{D}^{2}\right)}{\left(p^{2}+m_{+}^{2}\right)^{2}\left(p^{2}+m_{-}^{2}\right)^{2}}+2 \frac{\mu^{2} p^{2}}{\left(p^{2}+m_{+}^{2}\right)^{2}\left(p^{2}+m_{-}^{2}\right)^{2}}+2 \frac{m_{D}^{2}}{3 p^{2}\left(p^{2}+m_{+}^{2}\right)\left(p^{2}+m_{-}^{2}\right)}\right),
\end{aligned}
$$

and

$$
\delta \tilde{Z}=-\frac{2}{3} g^{2} \int \frac{d^{3} p}{(2 \pi)^{3}} \frac{\left(p^{2}+m_{D}^{2}\right)}{p^{2}\left(p^{2}+m_{+}^{2}\right)\left(p^{2}+m_{-}^{2}\right)} .
$$

To these, we add the Pisarski-Rao contributions (coming from the unbroken $S U(2)$ sector), yielding:

$$
Z_{m}=1+\frac{7 g^{2}}{6 \pi m}+\delta Z_{m}, \quad \tilde{Z}=1-\frac{g^{2}}{3 \pi m}+\delta \tilde{Z}
$$

which, in (9), yields

$$
q_{r e n}^{S U(2)}=q+2+q\left(\delta Z_{m}+2 \delta \tilde{Z}\right) .
$$

The remaining integrals are straightforward; one finds that $\delta Z_{m}+2 \delta \tilde{Z}=0$, and

$$
q_{r e n}^{S U(2)}=q+2,
$$

in agreement with [17]. 
Some comments are in order. First, $q_{r e n}^{S U(2)}$ is quantized, as it must be for consistency of the theory.' Second, there is nonetheless some peculiar behaviour exhibited. On the one hand, our final result (14) is completely independent of the expectation value of the scalar field, while on the other hand if we were to redo the entire calculation in the symmetric phase (in the absence of spontaneous symmetry breaking), $q_{S U(2)}$ would attain a renormalization exactly as in the pure gauge theory with the full gauge group $S U(3)$ : one would find $q_{r e n}^{S U(2)}=$ $q+3$.2 The limit of symmetry restoration in the above calculation must be examined with care: we must consider how the limit affects the integrals (10) and (11) rather than merely studying the final result (14). In fact, the problem can be traced to the third and fifth terms in the integrand of (10). For instance, the fifth term is simply not present in the symmetric phase since there is no gluon-gluon-scalar vertex (the vertex is proportional to $v$, whose presence in the fifth term is contained in $\left.m_{D}\right)$, while the symmetric limit $(v \rightarrow 0)$ of the integral of this term is nonzero. This ambiguity is due to an infrared problem which appears in the integrand as $v \rightarrow 0$. In this limit, $m_{-} \rightarrow 0$, and the integral is linearly divergent. That term's contribution to $\delta Z_{m}$ is of the order $g^{2} m_{D}^{2} / m_{-}$. Since $m_{D} \propto v$ while as $v \rightarrow 0$ $m_{-} \sim v^{2}$ (as can be seen from (8)), the contribution of that term is finite and nonzero as $v \rightarrow 0$, in disagreement with the zero result one would have obtained obtained in the symmetric theory. Similar considerations apply to the third term, while the other terms in (10) and (11) are well-behaved in the symmetric limit. Thus, we conclude that evaluating the integral and taking the limit of no symmetry breaking do not commute. Non-commutativity of limits has also been observed in perturbative calculations in CS theories in several other situations [13, 14, 19].

\footnotetext{
${ }^{1}$ It is perhaps worth reiterating that computational errors in the original version of this paper led us to a different conclusion, namely, that $q_{r e n}^{S U(2)}$ is not quantized.

${ }^{2}$ Note that, even though the symmetry is not broken to $S U(2)$ here, we are free to calculate the radiative correction to $q$ for the gluons of an $S U(2)$ subgroup.
} 
The calculations outlined above can be easily modified to handle other cases. We have studied the following patterns of symmetry breaking: $S U(2) \rightarrow U(1)$ via a real triplet which attains an expectation value $\left\langle\phi_{a}\right\rangle=v \delta_{a, 3} ; S U(3) \rightarrow S U(2) \times U(1)$ via an adjoint which attains an expectation value $\langle\Phi\rangle=v T_{8} \sim \operatorname{diag}(1,1,-2) ; S U(3) \rightarrow U(1) \times U(1)$ via an adjoint which attains an expectation value $\langle\Phi\rangle=v T_{3} \sim \operatorname{diag}(1,-1,0)$. In all cases, the Feynman rules are found in a straightforward way, and the diagrams which contribute to $Z_{m}$ and to $\tilde{Z}$ for an unbroken gluon are identical to those as calculated above. Differences arise only in the values of coupling constants, masses, and group theoretical factors. The calculation of the $Z \mathrm{~s}$ naturally separates into contributions from the unbroken group and possible contributions from the orthogonal (broken) sector. In the case of an unbroken group which is a direct product, $\delta q$ can be computed for each subgroup of the direct product. Straightforward group theoretical factors imply that the subgroups decouple: each subgroup of the unbroken group only contributes to its own $\delta q$. As for the contribution from the broken sector, in all cases it was found that $\delta Z_{m}+2 \delta \tilde{Z}=0$, and the net result was that $\delta q$ is that value one would have calculated from the pure gauge theory of the unbroken subgroup under consideration. Thus, in all cases the correction to $q_{U(1)}$ was zero, in keeping with expectations based on the Coleman-Hill theorem [16]. Furthermore, in the second case, the unbroken $S U(2)$ correction is as above: $\delta q_{S U(2)}=2$. The generalization is clear: the $q$ of any residual $U(1)$ symmetry receives no radiative correction, while that of any residual non-abelian group receives a radiative correction which is as if the orthogonal sector of the theory was not there.

We thank A.S. Goldhaber, M. Leblanc, G. W. Semenoff and V. P. Spiridonov for useful discussions. We are particularly indebted to G. Dunne for discussions of his results which enabled us to locate a couple of errors in the original version of this work. A. Khare thanks the Laboratoire de Physique Nucléaire for the kind invitation and hospitality during his visit. This work supported in part by NSERC of Canada and FCAR du Québec. 


\section{REFERENCES}

[1] W. Siegel, Nucl. Phys. B156, 135 (1979); J. Schonfeld, Nucl. Phys. B185, 157 (1981); R. Jackiw and S. Templeton, Phys. Rev. D 23, 2291 (1981).

[2] S. Deser, R. Jackiw and S. Templeton, Ann. Phys. (N.Y.) 140, 372 (1982).

[3] J. M. Leinaas and J. Myrheim, Nuovo Cimento 37B, 1 (1977); F. Wilczek, Phys. Rev. Lett. 48, 1144 (1982).

[4] D.P. Arovas, et al., Nucl. Phys. B251 [FS13], 117 (1985); F. Wilczek and A. Zee, Phys. Rev. Lett. 51, 2250 (1983).

[5] R. Laughlin, Phys. Rev. Lett. 50, 1395 (1983); B.I. Halperin, Phys. Rev. Lett. 52, 1583 (1984); S. C. Zhang, T. H. Hansson and S. Kivelson, Phys. Rev. Lett. 62, 82 (1989), and references therein.

[6] R. Laughlin, Science 242, 525 (1988); Y. H. Chen, B.I. Halperin, F. Wilczek and E. Witten, Int. Jour. Mod. Phys. B3, 1001 (1989).

[7] C.R. Hagen, Ann. Phys. (N.Y.) 157, 342 (1984).

[8] E. Witten, Comm. Math. Phys. 121, 351 (1989).

[9] E. Witten, Nucl. Phys. B311, 46 (1988).

[10] S. Weinberg, in "Understanding the Fundamental Constituents of Matter", editor A. Zichichi (Plenum Press, NY, 1978); A. Linde, Rep. Prog. Phys. 42, 389 (1979).

[11] R. D. Pisarski and S. Rao, Phys. Rev. D 32, 2081 (1985).

[12] G. Giavirini, C. P. Martin and F. Ruiz Ruiz, Nucl. Phys. B381, 222, 1992.

[13] S. Yu. Khlebnikov and M. E. Shaposhnikov, Phys. Lett. 254B, 148 (1991).

[14] S. Yu. Khlebnikov, JETP Letters 51, 81 (1990) V. P. Spiridonov, JETP Letters 52, $1112(1990)$. 
[15] A. Khare, R. MacKenzie and M.B. Paranjape, Phys. Lett. B343, 239-243 (1995).

[16] S. Coleman and B. Hill, Phys. Lett. 159B, 184 (1985); see also the discussion in [11].

[17] L. Chen, G. Dunne, K. Haller and E. Lim-Lombridas, Phys. Lett. 348B, 468 (1995).

[18] S. K. Paul and A. Khare, Phys. Lett. 171B, 244 (1986).

[19] M. Leblanc and M. T. Thomaz, Phys. Lett. 281B, 259 (1992). 